\title{
Impact of dislocations in monolithic III-V lasers on silicon: a theoretical approach
}

Hantschmann, Constanze, Liu, Zizhuo, Tang, Mingchu, Seeds, Alwyn, Liu, Huiyun, et al.

Constanze Hantschmann, Zizhuo Liu, Mingchu C. Tang, Alwyn J. Seeds, Huiyun Liu, lan H. White, Richard V. Penty, "Impact of dislocations in monolithic III-V lasers on silicon: a theoretical approach," Proc. SPIE 11274, Physics and Simulation of Optoelectronic Devices XXVIII, 112740J (2 March 2020); doi: $10.1117 / 12.2547327$

SPIE. Event: SPIE OPTO, 2020, San Francisco, California, United States 


\title{
Impact of Dislocations in Monolithic III-V Lasers on Silicon: A Theoretical Approach
}

\author{
Constanze Hantschmann*a, Zizhuo Liu ${ }^{\mathrm{b}}$, Mingchu C. Tang ${ }^{\mathrm{b}}$, Alwyn J. Seeds ${ }^{\mathrm{b}}$, Huiyun Liu ${ }^{\mathrm{b}}$, Ian H. \\ White ${ }^{\mathrm{a}, \mathrm{c}}$, and Richard V. Penty ${ }^{\mathrm{a}}$ \\ ${ }^{a}$ Centre for Photonic Systems, Department of Engineering, University of Cambridge, 9 JJ Thomson \\ Avenue, Cambridge CB3 OFA, UK \\ ${ }^{\mathrm{b}}$ Department of Electronic and Electrical Engineering, University College London, WC1E 7JE, UK \\ ${ }^{\mathrm{c}}$ University of Bath, Bath BA2 7AY, UK
}

\begin{abstract}
The growth of reliable III-V quantum well (QW) lasers on silicon remains a challenge as yet unmastered due to the issue of carrier migration into dislocations. We have recently compared the functionality of quantum dots (QDs) and QWs in the presence of high dislocation densities using rate equation travelling-wave simulations, which were based on $10-\mu \mathrm{m}$ large spatial steps, and thus only allowed the use of effective laser parameters to model the performance degradation resulting from dislocation-induced carrier loss. Here we increase the resolution to the sub-micrometer level to enable the spatially resolved simulation of individual dislocations placed along the longitudinal cavity direction in order to study the physical mechanisms behind the characteristics of monolithic $980 \mathrm{~nm} \mathrm{In}(\mathrm{Ga}) \mathrm{As} / \mathrm{GaAs}$ QW and $1.3 \mu \mathrm{m}$ QD lasers on silicon. Our simulations point out the role of diffusion-assisted carrier loss, which enables carrier migration into defect states resulting in highly absorptive regions over several micrometers in QW structures, whereas QD active regions with their efficient carrier capture and hence naturally reduced diffusion length show a higher immunity to defects. An additional interesting finding not accessible in a lower-resolution approach is that areas of locally reduced gain need to be compensated for in dislocation-free regions, which may lead to increased gain compression effects in silicon-based QD lasers with limited modal gain.
\end{abstract}

Keywords: Photonic integration, quantum dot lasers, quantum well lasers, semiconductor defects, semiconductor laser modelling, silicon photonics

\section{INTRODUCTION}

The monolithic integration of III-V lasers on silicon finds wide popularity due to the prospect of merging high optical gain materials with silicon-based electronics. Historically, progress on monolithic silicon photonic circuits was, however, hampered by the challenge of growing high-crystal-quality III-V layers on silicon substrates as a result of their different lattice constants, thermal expansion coefficients, and crystal polarities ${ }^{1,2}$. Numerous attempts have been made to grow GaAs-based $850 \mathrm{~nm}$ and $980 \mathrm{~nm}$ quantum well (QW) lasers directly on silicon, yet the resulting devices suffered mostly from poor lifetimes ${ }^{3,4}$ and high threshold current densities despite moderate dislocation densities of the order of $10^{6} \mathrm{~cm}^{-2}$ to $10^{7} \mathrm{~cm}^{-25}$,6. In the past decade, InAs/GaAs quantum dots (QDs) have instead established themselves as the gain medium of choice due to their higher tolerance to defects, having demonstrated lasing up to dislocation densities of $3 \times 10^{8} \mathrm{~cm}^{-27}$, which is an astounding four to five orders of magnitude higher than in commercial semiconductor lasers ${ }^{2}$. Yet despite considerable advances in the optimization of defect filter and buffer layers, none of the groups growing high-performance $1.3 \mu \mathrm{m}$ QD lasers on silicon have succeeded in demonstrating a silicon-based QW laser ${ }^{8,9}$. While few experimental studies exploring the nature of defects in InAs QD lasers on silicon have started to become available ${ }^{10,11}$, there is still a lack of theoretical work investigating the performance discrepancies between QD and QW lasers on silicon and how these lasers can be modelled appropriately. Here, we present an approach to simulating such devices using a rate equation travelling-wave model with high spatial resolution enabling the inclusion of individual dislocations. By studying the impact of dislocations in the laser active region, we aim to improve the understanding of the degrading performance typically observed from dislocation densities of about $10^{6} \mathrm{~cm}^{-2}$, focusing particularly on the limitations QW structures experience.

*cb893@cam.ac.uk; phone +44 1223 748363; www-g.eng.cam.ac.uk/photonic_comms 


\section{THEORETICAL MODEL}

Numerical simulations are performed using an excitonic multi-level rate equation travelling-wave model offering onedimensional spatial resolution along the longitudinal direction of the laser. The QD laser's energy structure is modelled as a coupled electronic system consisting of barrier layer, wetting layer and two discrete QD states, i.e. the ground state and excited state, respectively ${ }^{12,13}$. Although the barrier layer is often neglected due to their very small impact on the QD dynamics, we choose to include it for consistency with the QW simulations and due to the importance of dislocationinduced carrier loss in the continuum states. The used simulation parameters are summarized in Table 1.

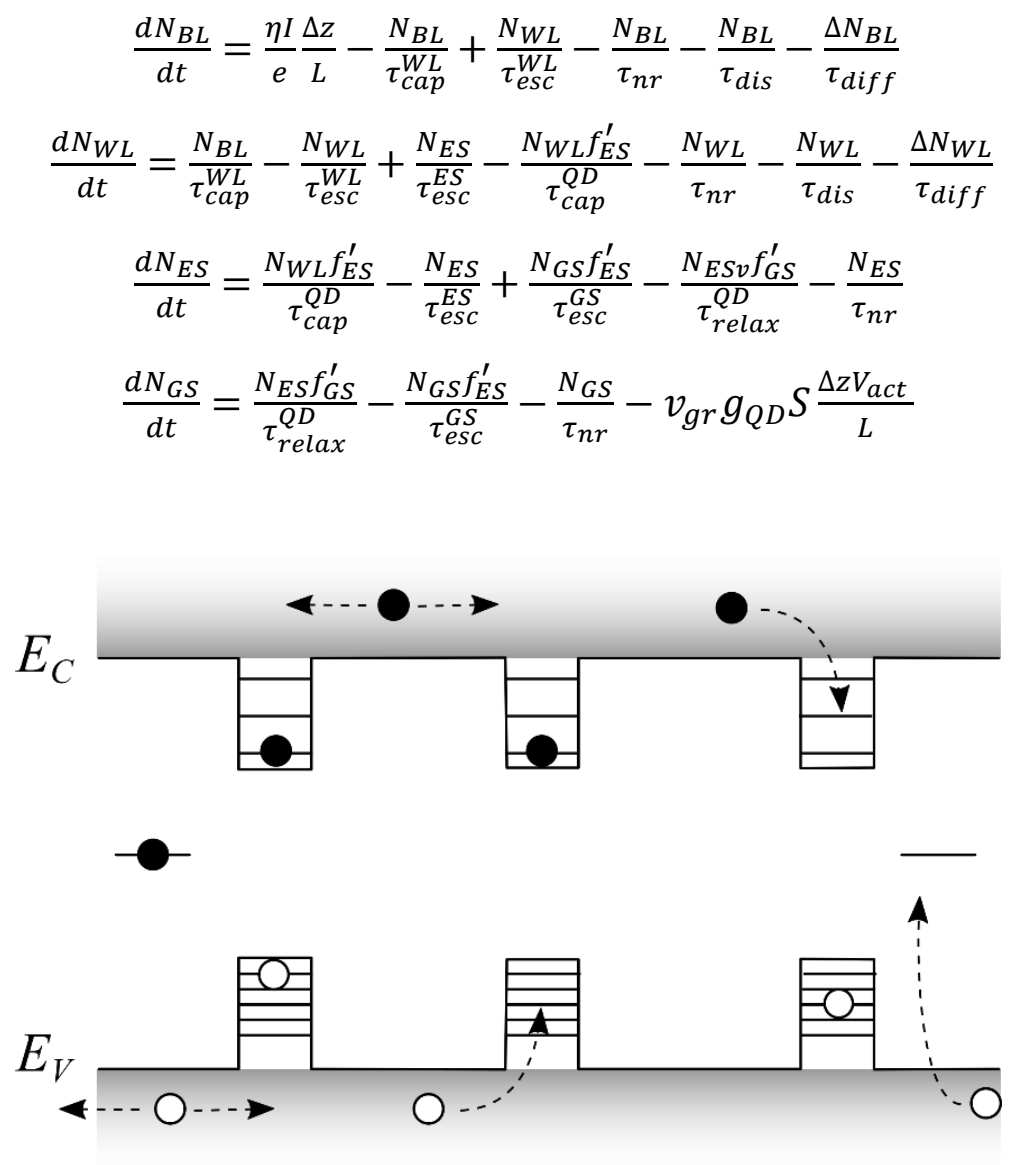

Figure 1. Schematic one-dimensional real-space energy band diagram illustrating the process of lateral carrier diffusion in the wetting layer of a QD active region and carrier capture into mid-bandgap nonradiative defect centers (dislocations). The average distance of about $50 \mathrm{~nm}$ between two QDs enables efficient carrier capture into the QDs.

The above equations for the calculation of the carrier numbers in each level describe the process of current injection into the barrier layer, from where they are captured into the wetting layer and relax in a cascaded process via the excited state into the QD ground state. At the same time, nonradiative carrier loss takes place in all levels and carriers escape through thermal excitation from the ground state, the excited state, and the wetting layer into higher energy level s. Lasing is considered from the ground state only. The scaling term $\Delta z V_{a c t} / L$ converts the photon density $S$ in the last term of Eq. (4) into a photon number in order to be consistent with the carrier numbers. Finally, lateral carrier diffusion is allowed in the barrier layer and wetting layer only, as the QDs are spatially isolated, as illustrated in Fig. 1. The respective time constant is calculated as $\tau_{\text {diff }}=\Delta z^{2} /(2 D)^{14}$, where the diffusion constant $D$ is obtained from a fixed diffusion length according to $L_{\text {diff }}=\sqrt{ }\left(D \tau_{n r}\right){ }^{15}$. Carrier diffusion lengths of several micrometers have been reported for GaAs-based QWs, whereas carrier diffusion in dot-in-a-well-based QD active regions is significantly reduced due to rapid carrier capture into the QDs ${ }^{16,17}$. This is naturally modelled in the above equations. Dislocation-induced carrier loss is considered via the new carrier 
Table 1. Overview of the used simulation parameters.

\begin{tabular}{|ll|}
\hline \multicolumn{2}{|c|}{ Common QW and QD Parameters } \\
\hline Cavity length $L=500 \mu \mathrm{m}$ & Injection efficiency $\eta=0.55$ \\
Waveguide width $w=2 \mu \mathrm{m}$ & Nonradiative lifetime $\tau_{n r}=1 \mathrm{~ns}$ (all levels) \\
Facet reflectivity $R_{l, 2}=0.95,0.30$ & QW/WL escape time $\tau_{e s c}^{Q W, W L}=2.2 \mathrm{ps}$ \\
Number of active layers $=5$ & Dislocation capture time $\tau_{\text {dis }}=10 \mathrm{ps}$ \\
Optical loss $\alpha_{i}=5 \mathrm{~cm}^{-1}$ & BL, QW/WL diffusion length $L_{\text {diff }}=10 \mu \mathrm{m}$ \\
& QD-Specific Parameters \\
\hline Wavelength $\lambda=1300 \mathrm{~nm}$ & Gain compression factor $\varepsilon=1 \times 10^{-16} \mathrm{~cm}^{3}$ \\
QD density $\rho=6 \times 10^{10} \mathrm{~cm}^{-2}$ & Ground state hole occupation $f_{G S}^{h}=0.5$ \\
Modal gain $g_{\text {mod }}=50 \mathrm{~cm}^{-1}$ & SCH transport $\&$ WL capture time $\tau_{c a p}^{W L}=3.4 \mathrm{ps}$ \\
Confinement factor $\Gamma=0.005$ & QD carrier capture time $\tau_{c a p}^{Q D}=3 \mathrm{ps}$ \\
SCH region thickness $=250 \mathrm{~nm}$ & QD relaxation time $\tau_{r e l a x}^{Q D}=0.5 \mathrm{ps}$ \\
WL thickness $=8 \mathrm{~nm}$ & GS, ES escape time $\tau_{\text {esc }}^{G S, E S}=2.7 \mathrm{ps,} 34 \mathrm{ps}$ \\
& QW-Specific Parameters \\
\hline Wavelength $\lambda=980 \mathrm{~nm}$ & Transparency carrier density $n_{t r}=1 \times 10^{18} \mathrm{~cm}^{-3}$ \\
Gain constant $g_{0}=3000 \mathrm{~cm}^{-1}$ & SCH region thickness $=65 \mathrm{~nm}$ \\
Confinement factor $\Gamma=0.05$ & QW thickness $=7.5 \mathrm{~nm}$ \\
Gain compression factor $\varepsilon=1 \times 10^{-17} \mathrm{~cm}^{3}$ & SCH transport \& QW capture time $\tau_{c a p}^{Q W}=3 \mathrm{ps}$ \\
\hline
\end{tabular}

loss rate $\tau_{d i s}{ }^{-1}$ included in the barrier and wetting layer, from where the carriers can migrate into nearby dislocations. Since carriers captured into QDs can only interact with dislocations in case they re-thermalize into the wetting layer, this term is omitted in Eqs. (3) and (4). Section III of this paper will further elaborate on the numerical implementation of threading dislocations. The barrier layer and wetting layer are both assumed to be a continuum of states, but in order to model the QD physics accurately, it is instructive to consider the two-fold and four-fold degeneracy of the QD ground state and excited state, respectively. The resulting Pauli blocking is included via occupation factors $f_{G S, E S}^{\prime}=\left(1-f_{G S, E S}\right)$, where $f_{G S, E S}$ is the occupation probability of the respective energy level ${ }^{18,19}$.

The QW laser is modelled analogously to the QD structure as a two-level electronic system consisting of the separate confinement heterostructure $(\mathrm{SCH})$ barrier layers and the QWs, but without the QD levels. As a consequence, the QW electron rate equations simplify to ${ }^{20,21}$

$$
\begin{gathered}
\frac{d N_{B L}}{d t}=\frac{\eta I}{e} \frac{\Delta z}{L}-\frac{N_{B L}}{\tau_{c a p}^{Q W}}+\frac{N_{Q W}}{\tau_{e s c}^{Q W}}-\frac{N_{B L}}{\tau_{n r}}-\frac{N_{B L}}{\tau_{d i s}}-\frac{\Delta N_{B L}}{\tau_{d i f f}} \\
\frac{d N_{Q W}}{d t}=\frac{N_{B L}}{\tau_{c a p}^{Q W}}-\frac{N_{Q W}}{\tau_{e s c}^{Q W}}-\frac{N_{Q W}}{\tau_{n r}}-\frac{N_{Q W}}{\tau_{d i s}}-\frac{\Delta N_{Q W}}{\tau_{\text {diff }}}-v_{g r} g_{Q W} S \frac{\Delta z V_{a c t}}{L} .
\end{gathered}
$$

The material gain functions $g_{Q D}$ and $g_{Q W}$ link the carrier occupation with the laser's optical properties via

$$
\begin{gathered}
g_{Q D}=g_{m a t}^{\max }\left(f_{G S}^{e}+f_{G S}^{h}-1\right) /(1+\varepsilon S) \\
g_{Q W}=g_{0} \ln \left({ }^{N} Q W / N_{t r}\right) /(1+\varepsilon S),
\end{gathered}
$$

where $g_{\text {mat }}^{\max }, f_{G S}^{e}, f_{G S}^{h}$, and $\varepsilon$ are the maximum QD material gain, the electron and hole ground state occupation probability, and the gain compression factor ${ }^{12}$, and $g_{0}$ and $N_{t r}$ are the gain constant and the carrier number per laser section at transparency ${ }^{21}$, respectively. 
The photon density is computed using a pair of travelling-wave electric field equations:

$$
\left(\frac{1}{v_{g r}} \frac{\partial}{\partial t} \pm \frac{\partial}{\partial z}\right) E^{ \pm}(z, t)=\left(\tilde{g}_{\text {mod }}-\tilde{\alpha}_{i}-i \delta\right) E^{ \pm}(z, t)+i_{s p \pm}(z, t) .
$$

Eq. (9) describes the slowly varying complex amplitudes of the forward and reverse propagation fields $E^{+}(z, t)$ and $E^{-}(z, t)$ excited by spontaneous noise $i_{s p} \pm$ and experiencing gain $\tilde{g}_{m o d}=1 / 2 g_{m o d}$, loss $\tilde{\alpha}_{i}=1 / 2 \alpha_{i}$, and phase detuning $\delta^{21}$. For numerical implementation, a Lorentzian filter is applied to model the wavelength dependence of the optical gain ${ }^{14}$. In a travelling-wave model, time and space steps $\Delta t$ and $\Delta z$ are connected via the group velocity as $\Delta z=v_{g r} \Delta t$. Many simulation environments allow comparably low spatial resolutions $(\sim 10 \mu \mathrm{m}$, for example), which is beneficial for computational speed, as the overall simulation time scales as $\Delta z^{-2}{ }^{14}$. The consequence is, however, that macroscopically averaged effective time constants and laser parameters have to be used to describe the almost homogeneous carrier and photon density. In the here presented approach, we aim to give a more accurate description of III-V lasers on silicon with high dislocation densities by increasing the spatial resolution to $\Delta z=500 \mathrm{~nm}$. This allows the explicit inclusion of nonradiative defect centers in the active region, as discussed in the next section.

\section{NUMERICAL IMPLEMENTATION OF DISLOCATIONS}

In our previous work, we have modelled the performance degradation with rising dislocation density $\rho_{d i s}$ by using a phenomenological carrier loss term for increasing dislocation-induced nonradiative recombination ${ }^{9}$. In this study, we take that approach a step further by positioning individual dislocations on a regular grid along the laser cavity, as illustrated in Fig. 2. The nonradiative recombination centers are modelled as laser sections $\Delta z$ with fast carrier loss rate $\tau_{\text {dis }}{ }^{-1}$, representing carrier capture into mid-bandgap defect states ${ }^{10}$. We set $\tau_{\text {dis }}$ to infinity in dislocation-free regions, while choosing a dislocation capture time $\tau_{\text {dis }}=10 \mathrm{ps}$ at $\Delta z=500 \mathrm{~nm}$ under the assumption of a carrier capture process of the order of a few picoseconds similar to the one into QD states. It should be noted that $\tau_{\text {dis }}$ is not only a fitting parameter due to the dislocation's unknown carrier capture time, but also as a result of the assumed dislocation core diameter and carrier capture length in conjunction with the chosen $\Delta z$. A variety of values has been published for the physical size of actual dislocation cores, ranging from below $1 \mathrm{~nm}^{22}$ over a few tens of nanometers ${ }^{15,23}$ up to $1 \mu \mathrm{m}^{24}$. The choice $\Delta z=500 \mathrm{~nm}$ is, accordingly, a trade-off between physical accuracy and computational feasibility. Selvidge et al. have recently reported a carrier capture length of $500 \mathrm{~nm}$ for InAs/GaAs QD lasers on silicon ${ }^{11}$, which supports our choice of spatial resolution.

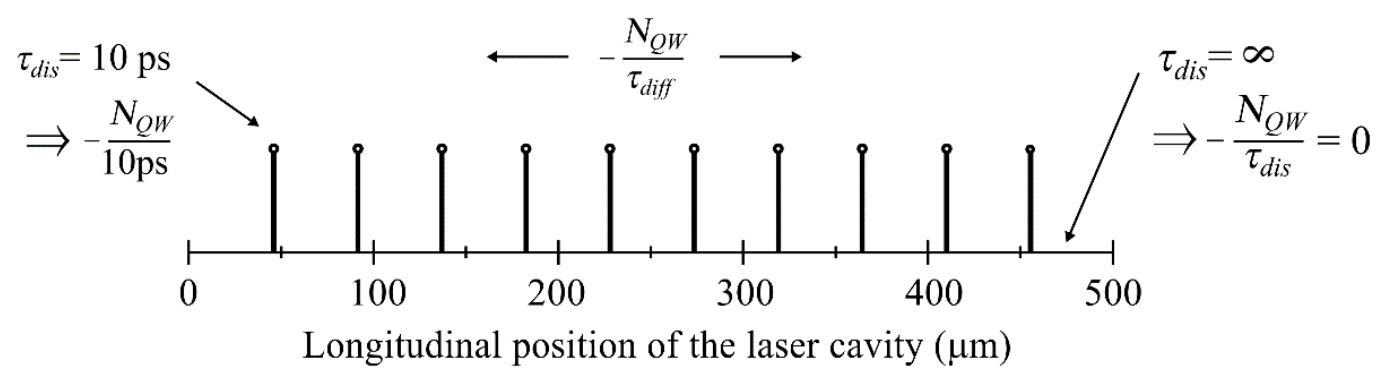

Figure 2. Schematic of the implementation of dislocation-induced carrier loss using a vector $\tau_{\text {dis }}$ with predefined dislocation positions given by the modelled dislocation density.

It should, furthermore, be noted that the here presented simulation approach is based on a few simplifications and assumptions, which will be discussed in the following. First of all, the travelling-wave approach neglects radial, i.e. two-dimensional carrier migration into dislocations, thus projecting this process onto a one-dimensional plane. Since this leads to an overestimation of the impact of dislocations on the laser performance, especially for QDs with their reduced effective diffusion length ${ }^{16,17}$, the waveguide width of the modelled devices is kept to $2 \mu \mathrm{m}$ at a maximum. This is additionally to ensure single-transverse mode operation. Secondly, as indicated in Fig. 2, the dislocations are arranged regularly along the cavity. Although a random dislocation distribution would correspond to a more realistic device scenario, the regular distribution is best in terms of result reproducibility. Simulations with different dislocation arrangements show that the respective laser performance variation is small. Lastly, dislocation-induced carrier loss is only included in the 
continuum energy levels, not in the QD states. A dislocation density of $10^{7} \mathrm{~cm}^{-2}$ amounts to only 100 threading dislocations in a $2 \times 500 \mu \mathrm{m}^{2}$ narrow ridge-waveguide laser, whereas the number of QDs in a $2 \times 0.5 \mu \mathrm{m}^{2}$ laser section containing five layers is 3,000 at a dot density of $6 \times 10 \mathrm{~cm}^{-2}$, for comparison. Hence, even for an optimistic dot coverage estimate of $10 \%{ }^{25}$, the number of individual dislocations hitting a $Q D$ is negligible.

\section{SIMULATION RESULTS AND DISCUSSION}

\subsection{Impact on the Carrier and Gain Distribution}

In order to understand the simulated light-current $(L I)$ performance of III-V lasers with different types of active regions in the presence of a high dislocation density, the behavior of the QD ground state and QW carrier densities as well as their respective gain profiles will be discussed first. Fig. 3(a) shows an example of the spatially resolved electron occupation probability of the QD ground state at about twice the threshold current for a threading dislocation density $\rho_{d i s}=1 \times 10^{6} \mathrm{~cm}^{-2}$, which equates to ten dislocations in the modelled device area of $2 \times 500 \mu \mathrm{m}^{2}$. For comparison, the dotted black line shows the ground state occupation probability at the same optical output power level in the absence of any dislocations. Clear dips where the ground state occupation probability falls below the threshold level can be seen due to reduced barrier layer and wetting layer carrier densities in the vicinity of a dislocation. Carrier diffusion intensifies this effect from the $500 \mathrm{~nm}$ long modelled dislocations to several micrometers, meaning that the occupation probability in dislocation-free regions must be increased to meet the required cavity threshold gain. The long minority carrier diffusion length in GaAs-based III-Vs, which is set to $10 \mu \mathrm{m}$ in these examples, contributes therefore to an increased sensitivity to defects ${ }^{26}$. Compared with the QW carrier density depicted in Fig. 3(b), it can be seen that the magnitude of the QD ground state occupation dip is actually relatively small, as ultrafast carrier capture into the dot states ${ }^{8}$ helps maintaining an overall high ground state occupation probability, even at reduced carrier densities in higher energy levels. Although exhibiting fundamentally the same features as the QD occupation in Fig. 3(a), the QW carrier distribution shows much deeper dips, because carriers accumulate in the QW layer rather than relaxing into deeper energy levels.

(a)

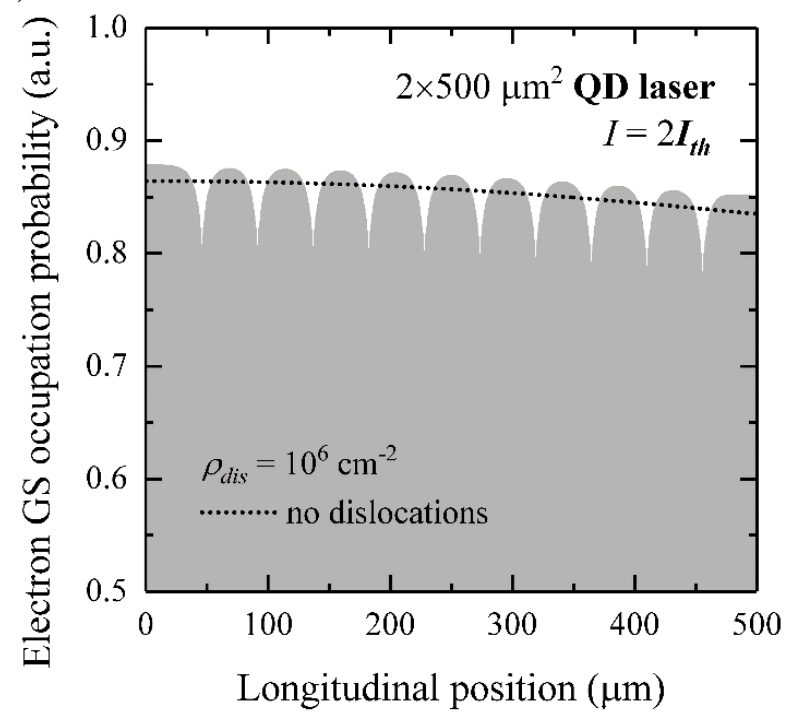

(b)

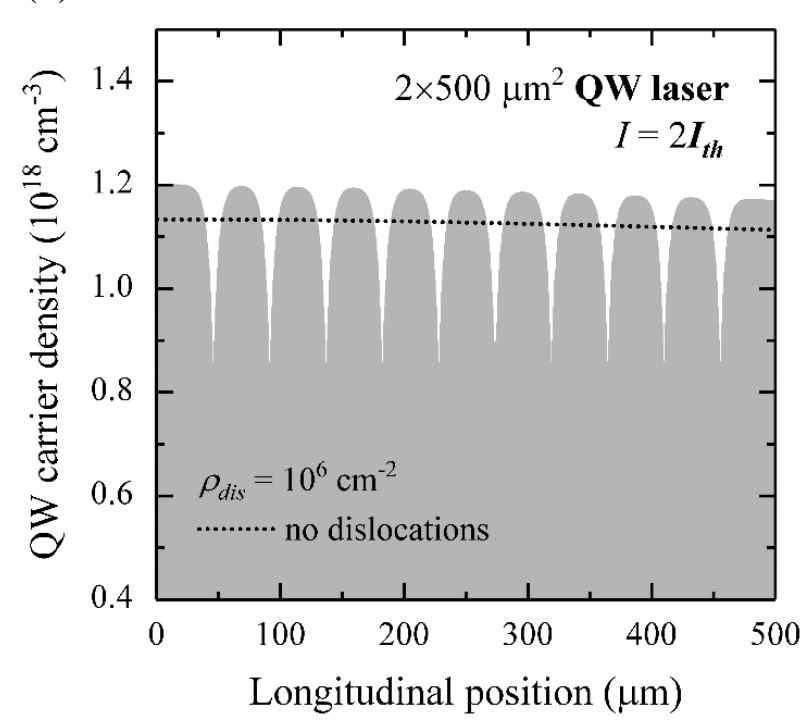

Figure 3. (a) QD ground state occupation probability and (b) QW carrier density lasers modelled using the parameters shown in Table 1. The dotted line indicates the respective values at the same optical output level in the absence of dislocations.

This behavior is directly reflected in the local gain profiles linking the laser's optical and electronic properties, as shown in Figs. 4(a) and (b). In accordance with the overall high occupation probability, the QD modal gain exhibits only a minor dip in the vicinity of a dislocation. In the case of the QW active region, in contrast, carrier-depleted areas around dislocations are highly absorptive due to the logarithmic nature of the QW gain function. It is only at higher current 
injection levels that dislocations begin to saturate ${ }^{27}$, as indicated in dark grey, so that diffusion-assisted carrier loss becomes less severe. It is interesting to note that the local gain between dislocations increases above the average threshold gain, which is indicated by the black dotted line, thus shifting the gain closer to its maximum modal gain. While this is less important for high-gain QW lasers, this effect may well increase gain compression effects in monolithic QD lasers on silicon, which are more prone to gain saturation due to their lower absolute modal gain ${ }^{28,29}$.

(a)

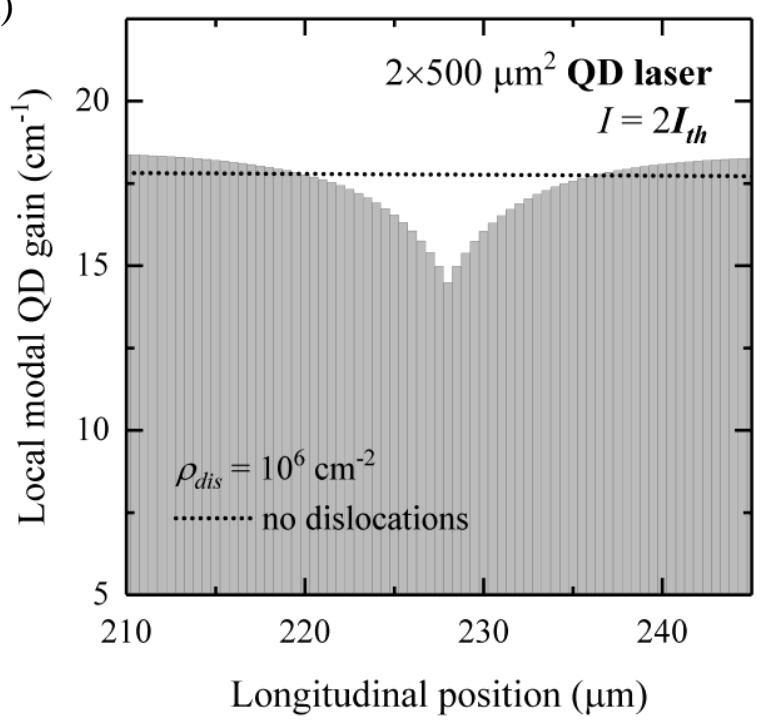

(b)

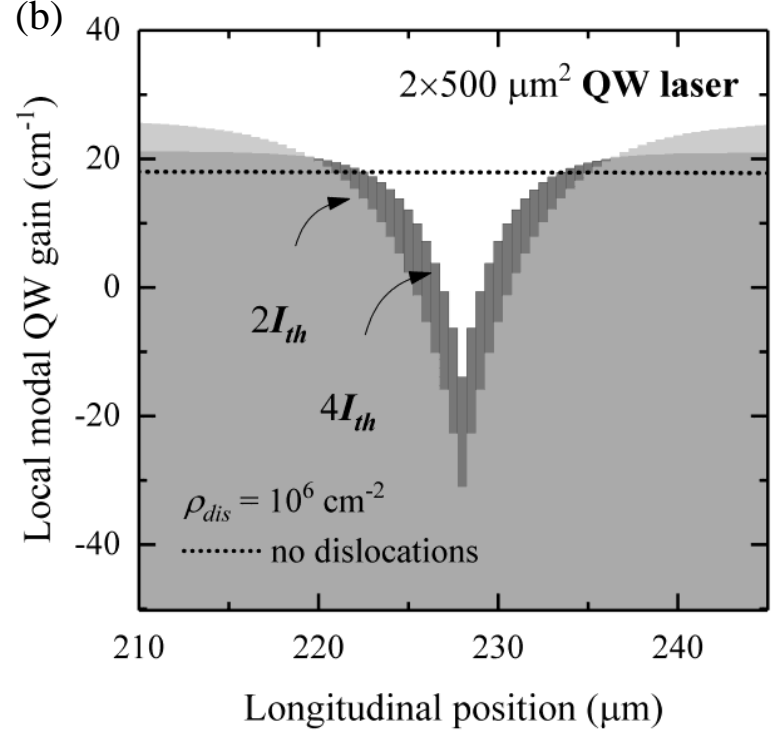

Figure 4. Insets of the gain functions corresponding to the carrier levels shown in Figs. 3(a) and (b) compared with the average gain without dislocations at the same optical output level. It should be noted that at the center of the dislocation core there is technically no QD or QW energy level providing gain at the respective wavelength. Due to the small dimensions of the crystal dislocation itself, this effect is, however, neglected.

\subsection{Light-Current Performance Trends}

The results of $L I$ simulations of $2 \times 500 \mu \mathrm{m}^{2} \mathrm{HR}$-coated QD and QW lasers at various threading dislocation densities can be seen in Figs. 5(a) and (b). The simulated QD $L I$ curves to the left confirm the experimentally observed trends of increased threshold currents and reduced slope efficiencies ${ }^{30,31}$, whereas the QW $L I$ curves to the right support the tendency of monolithic QW lasers on silicon to have higher threshold current densities ${ }^{5,6,9}$. One well-known factor causing high threshold currents is a short carrier lifetime ${ }^{19}$, as would be expected from simulations with high dislocation densities. From the direct comparison of Figs. 5(a) and (b) it becomes, however, evident that the modelled QD laser suffers from a much smaller threshold current increase than the QW laser. The key reason for this is that carrier diffusion in QD active regions is naturally reduced due to rapid carrier into QDs, where the spatially confined carriers remain isolated from nearby dislocations ${ }^{16}$. QW structures, on the contrary, allow diffusion-assisted carrier loss in both the QWs and the barrier layers within a radius of several micrometers around a dislocation, so that it takes QWs a much lower dislocation density to reduce their performance to a critical level. The QW laser's sensitivity to defects decreases accordingly with reduced minority carrier diffusion length. Simulations using a diffusion length of $1 \mu \mathrm{m}$, for example, show that the threshold current increase above $100 \mathrm{~mA}$ can be shifted from $\rho_{\text {dis }} \approx(3-4) \times 10^{6} \mathrm{~cm}^{-2}$ to about $8 \times 10^{6} \mathrm{~cm}^{-2}$, which may be sufficient for stateof-the-art III-V-on-Si growth technology. One possible approach for reduced in-plane diffusion could be offered by gaincoupled distributed-feedback (DFB) QW lasers, where a first-order gain grating of period $\Lambda=137 \mathrm{~nm}$ decreases the effective diffusion length to about $1.4 \mu \mathrm{m}$. Another option worth exploring is the utilization of InP, which shows reduced carrier diffusion (but is also associated with a larger lattice constant mismatch) ${ }^{1}$.

The $L I$ slope reduction observed in for the QD simulations results effectively from a reduced current injection efficiency due to excess dislocation-induced carrier loss in the continuum states. The QD $L I$ slope decreases substantially from $\rho_{\text {dis }} \gtrsim 10^{7} \mathrm{~cm}^{-2}$, where the wetting layer and barrier layer carrier densities drop to a level that begins to make carrier capture into the QD states problematic. This effect is dominant in QD lasers, which allow lasing at higher dislocation densities in 
the first place. The second contribution to the slope reduction stems from the reduction in local gain around dislocations, as the electric field intensity experiences a slight drop in these regions. It can, therefore, be understood intuitively that photons travelling along a laser cavity with many dislocations experience less amplification than photons in the presence of only a few dislocation-induced absorptive regions. Although this effect is primarily prevalent in QWs with their highly absorptive regions around dislocations, it can largely be compensated by high gain in dislocation-free regions and is practically overshadowed by the rapid threshold current increase. Despite the field intensity dips being less observed in QDs, it should be noted that QDs with their lower modal gain may be less able to compensate for locally reduced gain.

(a)

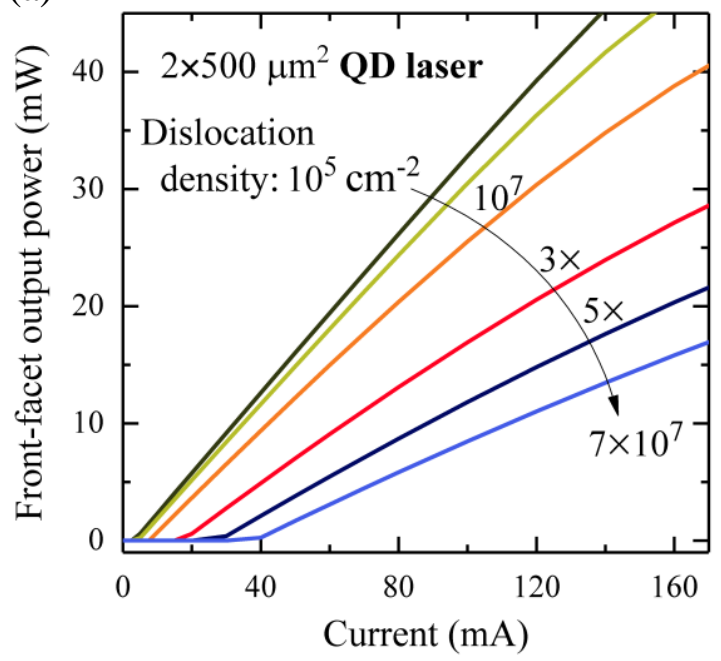

(b)

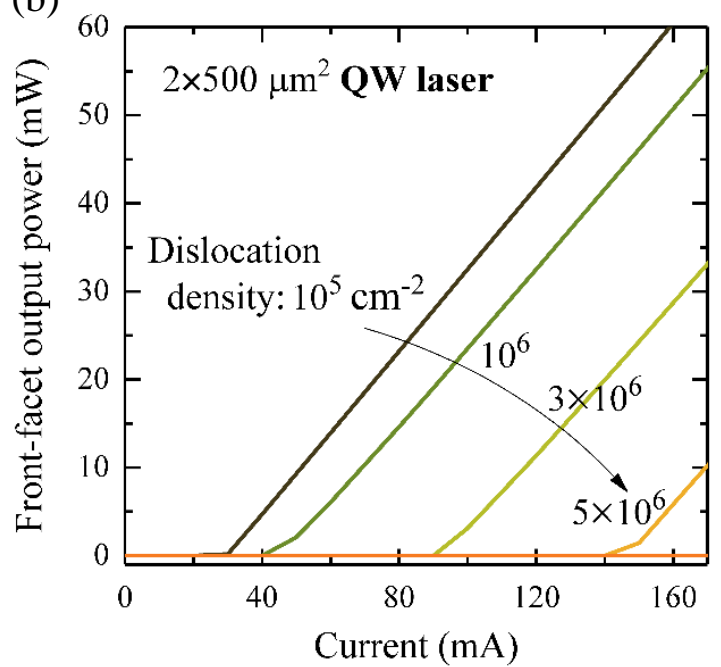

Figure 5. Modelled LI curves of an (a) QD and (b) QW laser with varying dislocation density.

\section{CONCLUSIONS AND OUTLOOK}

We have theoretically analyzed the effect of individual dislocations placed along the laser cavity on the performance of monolithic $\operatorname{In}(\mathrm{Ga}) \mathrm{As} / \mathrm{GaAs}$ QW and QD lasers on silicon substrate. Simulations based on a rate equation travelling-wave model with high spatial resolution are able to reproduce experimental trends such as threshold current increase and slope efficiency reduction demonstrated with both QD and QW devices on silicon, hence giving insights into the underlying laser physics under the impact of dislocations. The analysis of the gain, carrier density, and photon density distributions show that both dislocation-induced carrier loss as well as locally reduced gain appear to contribute to the observed laser characteristics of III-V lasers on silicon. Our high-resolution approach reveals not only that these gain dips inevitably have to be compensated for in dislocation-free regions, which may cause additional gain compression effects, but points also to the key role of diffusion-assisted carrier loss. Based on the fact that carrier migration into nearby dislocations affects QW active regions more strongly than their QD counterparts, we further identify gain-coupled QW DFB lasers with reduced defect sensitivity as a potential candidate to pave the way towards monolithically integrated high-gain QW lasers on silicon. It is noteworthy that in practice longer wavelength devices such as $1.3 \mu \mathrm{m} \mathrm{InAs} / \mathrm{GaAs}$ QDs or $1.3 \mu \mathrm{m}$ and $1.5 \mu \mathrm{m}$ InP-based QW lasers will be preferable for silicon photonics. Since QD lasers will, however, also require longer cavities due to their lower modal gain, this will limit direct modulation applications as well as the achievable integration density on the silicon photonics platform. So despite the current technological advantage monolithic III-V QD lasers on silicon hold over their QW-based counterparts, the ultimate decision on which technology to choose will, consequently, depend on several factors.

\section{ACKNOWLEDGEMENTS}

This project is in part funded by the UK EPSRC. C. Hantschmann wishes to thank Qualcomm Inc. for PhD funding as well as MKS Instruments for the SPIE Student Travel Grant. 


\section{REFERENCES}

[1] Dupuis, R. D., and Pinzone, C. J., "The growth of AlGaAs-GaAs lasers on Si substrates by metalorganic chemical vapor deposition," J. Cryst. Growth 93(1-4), 434-442 (1988).

[2] Liang, D., and Bowers, J. E., "Recent progress in lasers on silicon," Nat. Photonics 4(8), 511-517 (2010).

[3] Groenert, M. E., Leitz, C. W., Pitera, A. J., Yang, V., Lee, H., Ram, R. J., and Fitzgerald, E. A., "Monolithic integration of room-temperature cw GaAs/AlGaAs lasers on Si substrates via relaxed graded GeSi buffer layers," J. Appl. Phys. 93(1), 362-367 (2003).

[4] Groenert, M. E., Pitera, A. J., Ram, R. J., and Fitzgerald, E. A., "Improved room-temperature continuous wave $\mathrm{GaAs} / \mathrm{AlGaAs}$ and $\mathrm{InGaAs} / \mathrm{GaAs} / \mathrm{AlGaAs}$ lasers fabricated on $\mathrm{Si}$ substrates via relaxed graded $\mathrm{Ge}_{\mathrm{x}} \mathrm{Si}_{1-\mathrm{x}}$ buffer layers," J. V. Sci. Technol. B 21(3), 1064-1069 (2003).

[5] Aleshkin, V. Y., Baidus, N. V., Dubinov, A. A., Fefelov, A. G., Krasilnik, Z. F., Kudryavtsev, K. E., Nekorkin, S. M., Novikov, A. V., Pavlov, D. A., Samartsev, I. V., Shorokhodov, E. V., Shaleev, M. V. Sushkov, A. A., Yablonski, A. N., Yunin, P. A., and Yurasov, D. V., "Monolithically integrated InGaAs/GaAs/AlGaAs quantum well laser grown by MOCVD on exact Ge/Si(001) substrate," Appl. Phys. Lett. 109(6), 061111 (2016).

[6] Kim, J.-H., Radhakrishnan, G., Nouhi, A., Liu, J. K., Lang, R. J., and Katz, J., "High-power AlGaAs/GaAs DH strip laser diodes on GaAs-on-Si prepared by migration-enhanced molecular beam epitaxy,” Jpn. J. Appl. Phys.. 28(5), 791-796 (1989).

[7] Liu, A. Y., Peters, J., Huang, X., Jung, D., Norman, J., Lee, M. L., Gossard, A. C., and Bowers, J. E., "Electrically pumped continuous-wave $1.3 \mu \mathrm{m}$ quantum-dot lasers epitaxially grown on on-axis (001) GaP/Si," Opt. Lett. 42(2), 338-341 (2017).

[8] Liu, A. Y., Srinivasan, S., Norman, J., Gossard, A. C., and Bowers, J. E., "Quantum dot lasers for silicon photonics," Photon Res. 3(5), B1-B9 (2015)

[9] Liu, Z., Hantschmann, C., Tang, M., Lu, Y., Park, J.-S., Liao, M., Pan, S., Sanchez, A., Beanland, R., Martin, M., Baron, T., Chen, S., Seeds, A., Penty, R., White, I., and Liu, H., "Origin of defect tolerance in InAs/GaAs quantum dot lasers grown on silicon," published online, DOI: 10.1109/JLT.2019.2925598 (2019).

[10] Huang, J., Wan, Y., Jung, D., Norman, J., Shang, Chen, Li, Q., Lau, K. M., Gossard, A. C., Bowers, J. E, and Chen, B., "Defect characterization of InAs/InGaAs quantum dot p-i-n photodetector grown on GaAs-on-Vgrooved-Si substrate," ACS Photonics 6(5), 1100-1105 (2019).

[11] Selvidge, J., Norman, J., Salmon, M. E., Hughes, E. T., Bowers, J. E., Herrick, R., and Mukherjee, K., "Nonradiative recombination at dislocations in InAs quantum dots grown on silicon," Appl. Phys. Lett. 115(13), 131102-1-131102-5 (2019).

[12] Hantschmann, C., Vasil'ev, P. P., Wonfor, A., Chen, S., Liao, M., Seeds, A. J., Liu, H., Penty, R. V., and White, I. H., "Understanding the bandwidth limitations in monolithic $1.3 \mu \mathrm{m} \mathrm{InAs} / \mathrm{GaAs}$ quantum dot lasers on silicon," J. Lightw. Technol. 37(3), 949-955 (2018).

[13] Gioannini, M., Sevega, A., and Montrosset, I., "Simulations of differential gain and linewidth enhancement factor of quantum dot semiconductor lasers," Opt. Quant. Electron. 38(4-6), 381-394 (2006).

[14] Thompson, M. G., "Ultra-short pulse generation in quantum well and quantum dot monolithic mode-locked laser diodes," Ph.D. dissertation, Department of Engineering, University of Cambridge, Cambridge, UK (2006).

[15] Chernyak, L., Osinsky, A., and Schulte, A., "Minority carrier transport in GaN and related materials," Solid-State Electron. 45(9), 1687-1702 (2001).

[16] Popescu, D. P., Eliseev, P. G., Stintz, A., and Malloy, K. J., "Carrier migration in structures with InAs quantum dots," J. Appl. Phys. 94(4), 2454-2458 (2003).

[17] Moore, S. A., O'Faolain, L., Catalun, M. A., Flynn, M. B., Kotlyar, M. V., and Krauss, T. F., "Reduced surface sidewall recombination and diffusion in quantum-dot lasers," IEEE Photon. Technol. Lett. 18(17), 1861-1863 (2006).

[18] Gioannini, M., and Rossetti, M., "Time-domain traveling wave model of quantum dot DFB lasers," IEEE J. Sel. Top. Quantum Electron. 17(5), 1318-1326 (2011).

[19]Fiore, A., and Markus, A., "Differential gain and gain compression in quantum-dot lasers," IEEE J. Quantum Electron. 43(3), 287-297 (2007).

[20] McDonald, D., and O'Dowd, R. F., "Comparison of two- and three-level rate equations in the modelling of quantum-well lasers," IEEE J. Quantum Electron. 31(11), 1927-1934 (1995).

[21] Williams, K. A., Thompson, M. G., and White, I. H., "Long-wavelength monolithic mode-locked diode lasers," New J. Phys. 6(1), 179 (2004). 
[22] Karpov, S. Y., and Makarov, Y. N., "Dislocation effect on light emission efficiency in gallium nitride," Appl. Phys. Lett. 82(25), 4721-4723 (2002).

[23] Sugahara, T., Sato, H., Hao, M., Naoi, Y., Kurai, S., Tottori, S., Yamashita, K., Nishino, K., Romano, L. T., and Sakai, S., "Direct evidence that dislocations are non-radiative recombination centers in GaN," Jpn. J. Appl. Phys. 37(4A), L398-L400 (1998).

[24] Donolato, C., "Modeling the effect of dislocations on the minority carrier diffusion length of a semiconductor," J. Appl. Phys. 84(5), 2656-2664 (1998).

[25] Kirstaedter, N., Schmidt, O. G., Ledentsov, N. N., Bimberg, D., Ustinov, V. M., Egorov, A. Y., Zhukov, A. E., Maximov, M. V., Kop'ev, P. S., and Alferov, Z. I., "Gain and differential gain of single layer InAs/GaAs quantum dot injection lasers," Appl. Phys. Lett. 69(9), 1226-1228 (1996).

[26] Andre, C. L., Boeckl, J. J., Wilt, D. M., Pitera, A. J., Lee, M. L., Fitzgerald, E. A., Keyes, B. M., and Ringel, S. A., "Impact of dislocations on minority carrier electron and hole lifetimes in $\mathrm{GaAs}$ grown on metamorphic $\mathrm{SiGe}$ substrates," Appl. Phys.. Lett. 84(18), 3447-3449 (2004).

[27]Liu, H. Y., Sellers, I. R., Badcock, T. J., Mowbray, D. J., Skolnick, M. S., Groom, K. M., Gutiérrez, M., Hopkinson, M., Ng, J. S., David, J. P. R., and Beanland, R. "Improved performance of $1.3 \mu \mathrm{m}$ InAs quantum-dot lasers using high-growth-temperature GaAs spacer layer," Appl. Phys. Lett. 85(5), 704-706 (2004).

[28] Grillot, F., Dagens, B., Provost, J.-G., Su, H., and Lester, L. F., "Gain compression and above-threshold linewidth enhancement factor in 1.3- $\mu \mathrm{m}$ InAs-GaAs quantum-dot lasers," IEEE J. Sel. Quantum Electron. 44(10), 946-951 (2008).

[29] Matthews, D. R., Summers, H. D., Smowton, P. M., and Hopkinson, M., "Experimental investigation of the effect of wetting-layer states on the gain-current characteristic of quantum-dot lasers," Appl. Phys. Lett. 81(26), 49044906 (2002).

[30] Jung D., Herrick, R., Norman, J., Turnlund, K., Jan, C., Feng, K., Gossard, A. C., and Bowers, J. E., "Impact of threading dislocation density on the lifetime of InAs quantum dot lasers on Si," Appl. Phys. Lett. 112(15), 1535071-153507- (2018).

[31] Orchard, J. R., Shutts, S., Sobiersierski, A., Wu, J., Tang, M., Chen, S., Jiang, Q., Elliott, S., Beanland, R., Liu, H., Smowton, P. M., and Mowbray, D. J., "In situ annealing enhancement of the optical properties and laser device performance of InAs quantum dots grown on Si substrates," Opt. Express 24(6), 6196-6202 (2016). 